\title{
Morphological, Pathogenic and Toxigenic Variability in the Rice Sheath Rot Pathogen Sarocladium Oryzae
}

\author{
Kaat José Peeters ${ }^{1}$, Ashley Haeck ${ }^{2}$, Lies Harinck ${ }^{2}$, Oluwatoyin Oluwakemi Afolabi ${ }^{1}$, \\ Kristof Demeestere ${ }^{2}$, Kris Audenaert ${ }^{3}(\mathbb{D})$ and Monica Höfte ${ }^{1, *}$ \\ 1 Laboratory of Phytopathology, Department of Plants and Crops, Faculty of Bioscience Engineering, \\ Ghent University, Coupure Links 653, 9000 Ghent, Belgium; kaat.peeters@ugent.be (K.J.P.); \\ afolabi_toy@yahoo.com (O.O.A.) \\ 2 Research Group EnVOC, Department of Green Chemistry and Technology, Ghent University, \\ Coupure Links 653, 9000 Ghent, Belgium; ashhaeck@hotmail.com (A.H.); lies.harinck@ugent.be (L.H.); \\ kristof.demeestere@ugent.be (K.D.) \\ 3 Laboratory of Applied Mycology and Phenomics, Department of Plants and Crops, \\ Faculty of Bioscience Engineering, Ghent University, Valentin Vaerwyckweg 1, 9000 Ghent, Belgium; \\ kris.audenaert@ugent.be \\ * Correspondence: monica.hofte@ugent.be; Tel.: +32-9264-6017
}

Received: 26 December 2019; Accepted: 5 February 2020; Published: 8 February 2020

\begin{abstract}
Sheath rot is an emerging rice disease that leads to considerable yield losses. The main causal agent is the fungus Sarocladium oryzae. This pathogen is known to produce the toxins cerulenin and helvolic acid, but their role in pathogenicity has not been clearly established. S. oryzea isolates from different rice-producing regions can be grouped into three phylogenetic lineages. When grown in vitro, isolates from these lineages differed in growth rate, colour and in the ability to form sectors. A diverse selection of isolates from Rwanda and Nigeria, representing these lineages, were used to further study their pathogenicity and toxin production. Liquid chromatography high-resolution mass spectrometry analysis was used to measure cerulenin and helvolic acid production in vitro and in planta. The three lineages clearly differed in pathogenicity on the japonica cultivar Kitaake. Isolates from the least pathogenic lineage produced the highest levels of cerulenin in vitro. Helvolic acid production was not correlated with the lineage. Sectorisation was observed in isolates from the two least pathogenic lineages and resulted in a loss of helvolic acid production. In planta, only the production of helvolic acid, but not of cerulenin, correlated strongly with disease severity. The most pathogenic isolates all belonged to one lineage. They were phenotypically stable, shown by the lack of sectorisation, and therefore maintained high helvolic acid production in planta.
\end{abstract}

Keywords: Oryza sativa; cerulenin; helvolic acid; sectorisation; phenotypic stability; LC-MS/MS; Africa

Key Contribution: The pathogenicity of S. oryzae on the rice sheath is correlated with in planta helvolic acid production.

\section{Introduction}

Rice is the main staple food for more than half of the world's population [1,2]. More than 500 million tons of milled rice were produced in 2017, of which 30,000 tons were from Africa [3]. Due to population growth, urbanisation and dietary changes, rice consumption in Africa grows at about $5.5 \%$ per year (2000-2010 average) [1,4]. As the demand for rice strongly exceeds the local production, $43 \%$ of the rice consumed in Africa is imported, which costs more than USD 1.5 billion per year $[5,6]$. There is, however, great potential to increase the rice yield in Africa by improving crop management 
and expanding rice production. The major constraints are abiotic stresses (drought or excess of water, nutrient deficiencies and extreme temperatures) and biotic stresses (weeds, diseases and pests) [4].

Sheath rot is considered one of the most important emerging diseases of rice, causing yield losses from $20-30 \%$ up to $85 \%$ [7-9]. This disease is mainly caused by the seed-borne fungus Sarocladium oryzae [10]. Since its first description in 1922 in Taiwan as Acrocylindrium oryzae, the fungus has spread worldwide. Sheath rot occurs in both rainfed and irrigated ecosystems, affecting all rice varieties. Dwarf and high-yield Asian varieties are the most susceptible. S. oryzae infection becomes visible on the flag leaf sheath as greyish-brown necrotic lesions, enlarging as the disease progresses until the whole leaf sheath is necrotic. Enclosed panicles are affected, leading to sterile, empty or discoloured grains or, during severe infection, only partial or no emergence of the panicle $[9,10]$. S. oryzae can survive in seeds, plant residues, weeds and soil and it is transmitted by seeds, wind and insects. Wounds and stomata are needed to enter the host $[7,10]$.

Cell wall degrading enzymes and the phytotoxins helvolic acid, cerulenin and SO-toxin are described to be the main pathogenicity factors used by S. oryzae [11,12]. Clear evidence for the role and importance of cerulenin and helvolic acid in the disease development of sheath rot, however, is still missing. Helvolic acid is a tetracyclic triterpenoid that is able to bind magnesium-ions, thereby interfering with chlorophyll biosynthesis, which leads to chlorosis in the host tissue $[13,14]$. This compound is structurally very similar to the well-known steroid antibiotic fusidic acid, produced by Fusidium coecineum. Both compounds are toxic to Gram-positive bacteria $[15,16]$. Helvolic acid is produced by various fungi including the entomopathogenic fungus Metarhizium anisopliae and the opportunistic human pathogen Aspergillus fumigatus [17]. Also, the hexaketide amide, cerulenin, is both phytotoxic and antimicrobial. This compound interferes with fatty acid and polyketide biosynthesis by inhibiting the malonyl-ACP:acyl-ACP condensation step [18]. Its antifungal activity against different fungi, among which is Sclerotium oryzae and Magnaporthe oryzae, is well described $[14,19,20]$. The production of secondary metabolites, with both phytotoxic and antimicrobial actions, improves the competitive ability of $S$. oryzae. Thereby its survival increases and, consequently, its pathogenic potential increases as well [14].

Secondary metabolite production is known to be favoured by suboptimal growth conditions, which reflect the natural environment. Here, fungi are subjected to different environmental stimuli such as carbon sources, reactive oxygen species and interspecies communication. These stimuli can induce secondary metabolism $[21,22]$. When, however, the level of stress exceeds the tolerance level of the fungus, culture degeneration can occur. In culture, this process can lead to the formation of morphological variants that are called sectorisation. Sectors can vary in their pigmentation and are often impaired in their ability to produce secondary metabolites, pathogenicity-related enzymes and spores [23-28]. Since the frequency of sector formation is negatively correlated with the stability of the isolate in the natural environment and its virulence, this process should be taken into account when studying pathogen populations $[23,26,28]$.

The occurrence of rice sheath rot can cause considerable yield losses, and effective control measures are not available yet. Because of the different causal agents of sheath rot, and the lack of knowledge about their mode of action and resistance factors, there are no resistant varieties $[8,12]$. Seed treatments with fungicides are not effective because they do not kill the fungi inside the glume [7,12]. Pseudomonas fluorescens has been studied as a possible biocontrol agent against $S$. oryzae but the data in the field are still inconsistent $[7,29]$. In order to find effective control measures against this disease, it is important to elucidate the mode of action of the causal agent and study the diversity of the $S$. oryzae population.

Previous diversity studies in India and Bangladesh [12,30] have described the morphological and genetic variations and the pathogenicity of the S. oryzae population in different agro-ecological regions. A study that includes isolates from different regions worldwide, however, is still missing. Also, the population of S. oryzae in Africa is poorly studied and the etiology of rice sheath rot still needs to be further investigated. Both cerulenin and helvolic acid production have been measured in in vitro cultures but, so far, the toxin production in the host plant has not yet been quantified $[13,14]$. 
Based on the partial sequences of the internal transcribed spacer and actin genes, S. oryzae can be divided into three lineages [31-33]. In this work, we studied these phylogenic groups, based on S. oryzae isolates from Rwanda and Nigeria together with S. oryzae isolates originating from different rice-producing countries worldwide. The morphological and pathogenic variability was studied together with the in vitro and in planta toxin production of these isolates. By studying the production of helvolic acid and cerulenin during the infection process of isolates with a different level of pathogenicity, we aim to elucidate the importance of these toxins in the infection process of the host.

\section{Results}

\subsection{Morphological Characterisation and Growth Rate}

Isolates from the three phylogenetic lineages of $S$. oryzae were grown on potato dextrose agar (PDA) and oatmeal agar (OA) for 15 days to study their morphology and growth rate. The radial growth rate was calculated based on the colony diameter at five time points (Figure 1). With an average radial growth rate of $1.1 \mathrm{~mm}^{- \text {day }^{-1}}$, isolates of Group 1 grew, except for CBS120.817 and CBS485.80, significantly slower on PDA than the isolates of Groups 2 and 3. The radial growth rate varied a lot (from 1.3 to 2.5 mm.day ${ }^{-1}$ ) among the isolates of Group 2, while Group 3 showed a more constant radial growth rate of meanly $1.8 \mathrm{~mm}$.day ${ }^{-1}$ (Figure $1 \mathrm{~A}, \mathrm{C}$ ). On oatmeal agar $(\mathrm{OA})$ the radial growth rate was more constant than on PDA (Figure 1A,B). When grown on OA, all three groups had a mean radial growth rate between 1.9 and $2.0 \mathrm{~mm} \cdot \mathrm{day}^{-1}$ (Figure 1C). Although the variation among the isolates of one group was higher than this difference of $0.1 \mathrm{~mm}$ day ${ }^{-1}$, the variation was still smaller on OA (Figure 1B).

As shown in Figure 2, the colony colour of the $S$. oryzae isolates, grown on PDA, varied between white, pale salmon and orange. The brightest coloured isolates belonged to Group 1 (RFRG2 and IBNG0013). The colonies grew powdery and were radially folded, pale to dark orange with a white border. Isolates of Group 2 had radially folded (RFNG33) or radially striated (RFBG9) white or pale salmon colonies. The mycelium growth varied from powdery to wet aerial. Colonies of Group 3 were morphologically the most uniform and the most distinct from the other groups. All isolates formed powdery, dark or pale orange colonies on PDA, and some isolates produced a deep yellow diffusible pigment (BDNG0025). Isolates of Groups 1 and 2 showed sectorisation on PDA. For the isolates of Group 3, no sector formation was observed. The sectors differed in their morphological characteristics such as growth rate and colour.

The Group 1 isolate CBS180.74 was used to further study sectorisation. Four different phenotypes were observed on PDA. Being the original and most abundant phenotype, the white phenotype was considered as the parent culture. This white phenotype showed a radially folded, powdery growth with a light salmon-green shade. On PDA, sectors formed in this culture in three different phenotypes. The phenotypes mainly differed in colour (brown, green, orange) and growth rate. Some phenotypes grew more powdery than the parent and all cultures were radially folded (Figure 2). 


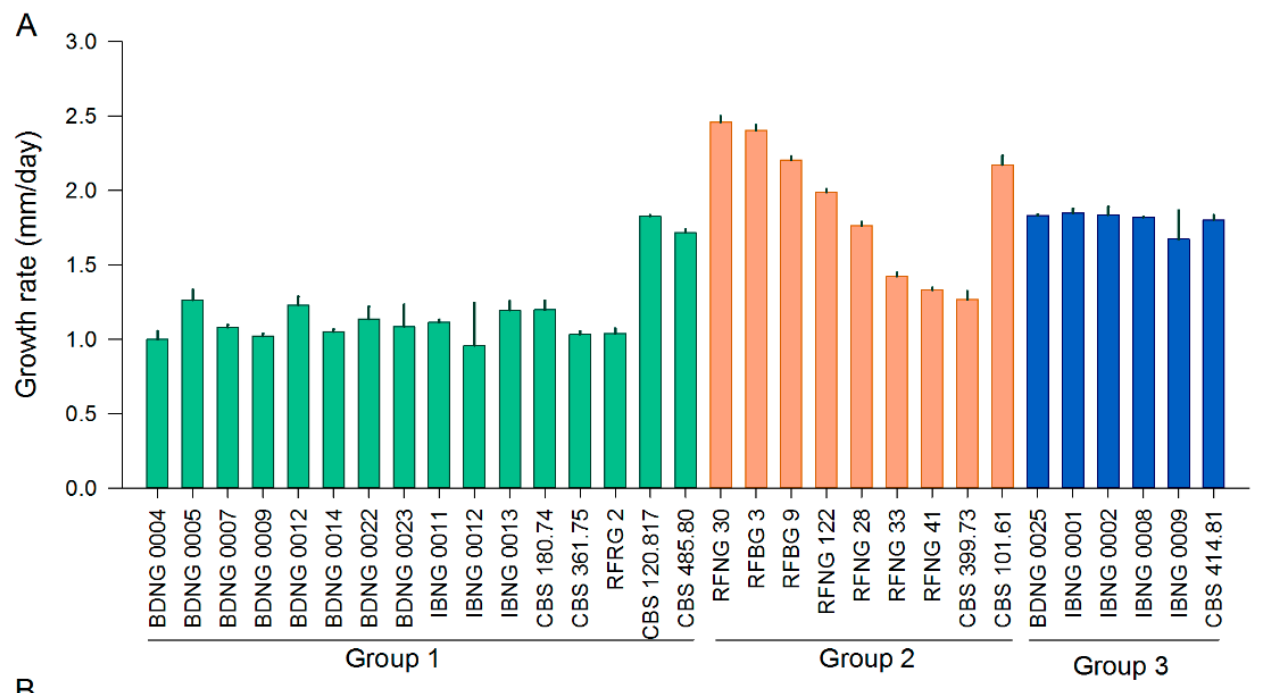

B

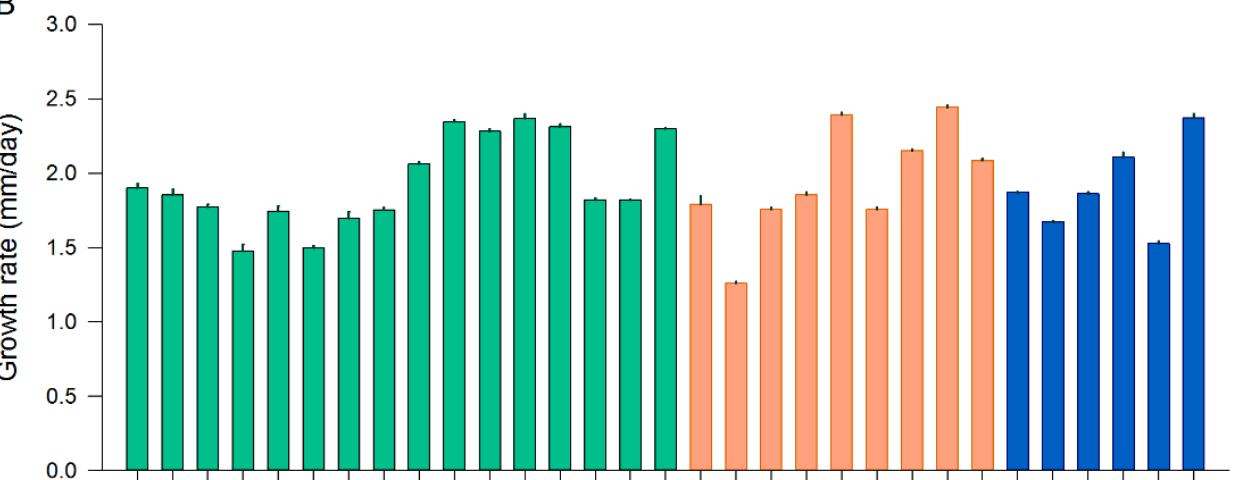

ఫั

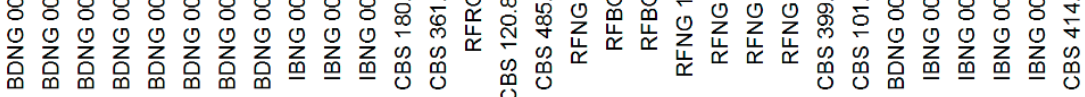
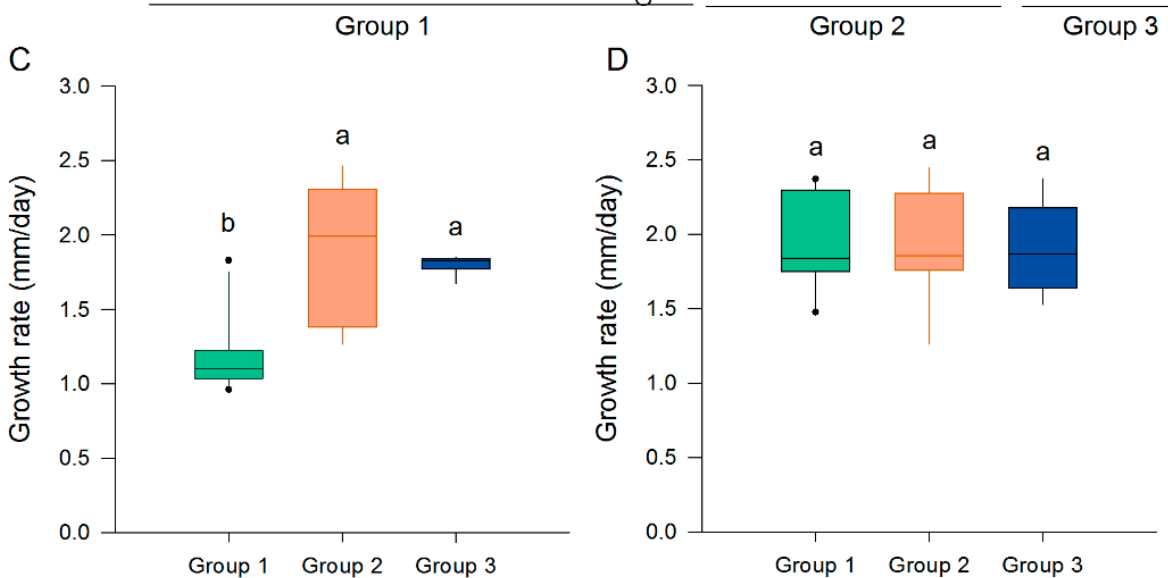

Figure 1. Radial growth rate of $S$. oryzae isolates grown on potato dextrose agar (PDA) or oatmeal agar (OA). (A) Radial growth rate of individual isolates on PDA. (B) Radial growth rate of individual isolates on OA. All bars show the mean \pm SE with three replicates for each isolate. (C) Radial growth rate of all isolates belonging to Groups 1, 2 and 3 on PDA. (D) Radial growth rate of all isolates belonging to Groups 1, 2 and 3 on OA. Isolates of Groups 1, 2 and 3 are represented by, respectively, green, orange and blue boxplots. Boxplots represent the median with the first and third quartile, the whiskers show the minimum and maximum values. Outliers and extreme values are represented by dots. Boxplots marked with different letters are statistically different (PDA: Mann-Whitney, $18 \leq n \leq 48, \alpha=0.05$; OA: ANOVA, $18 \leq n \leq 48, \alpha=0.05$ ). 

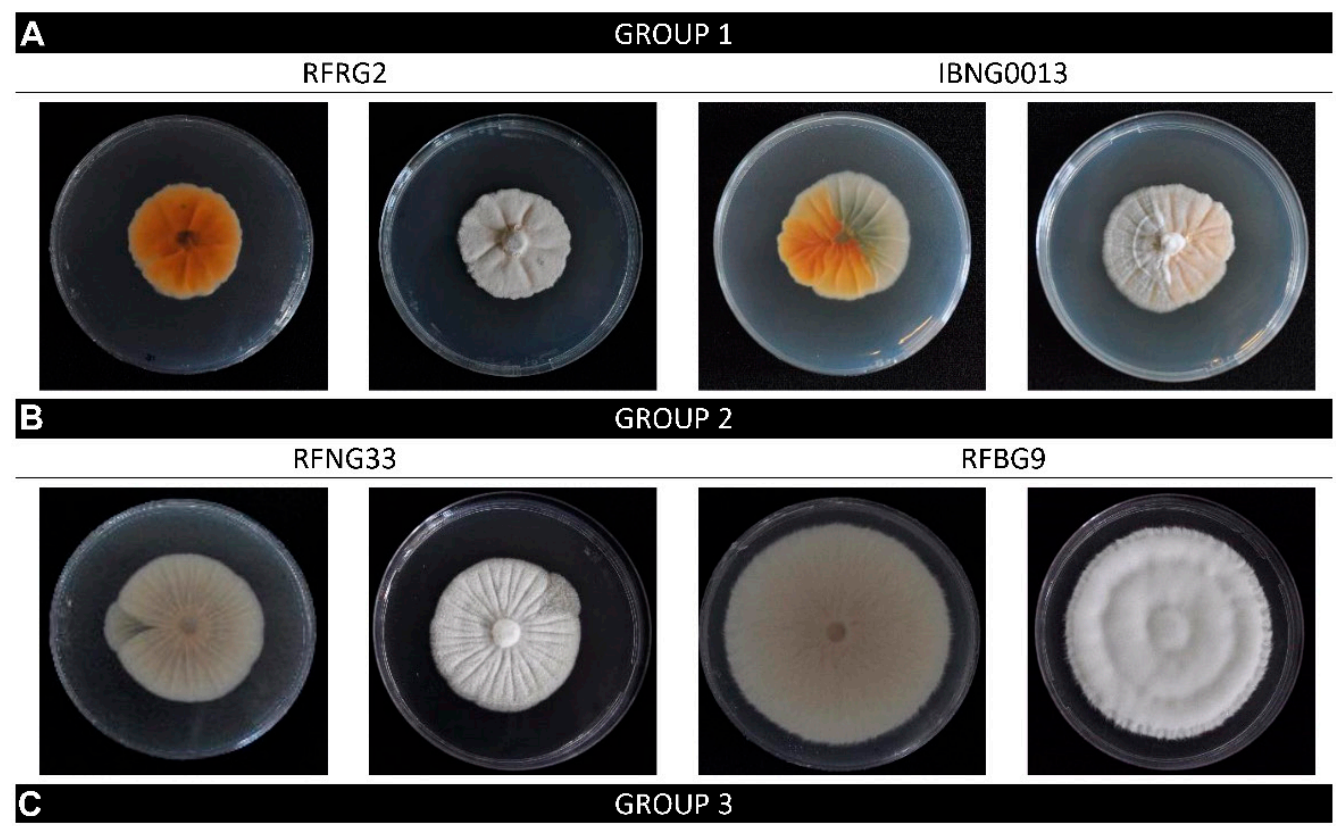

C

BDNG0025
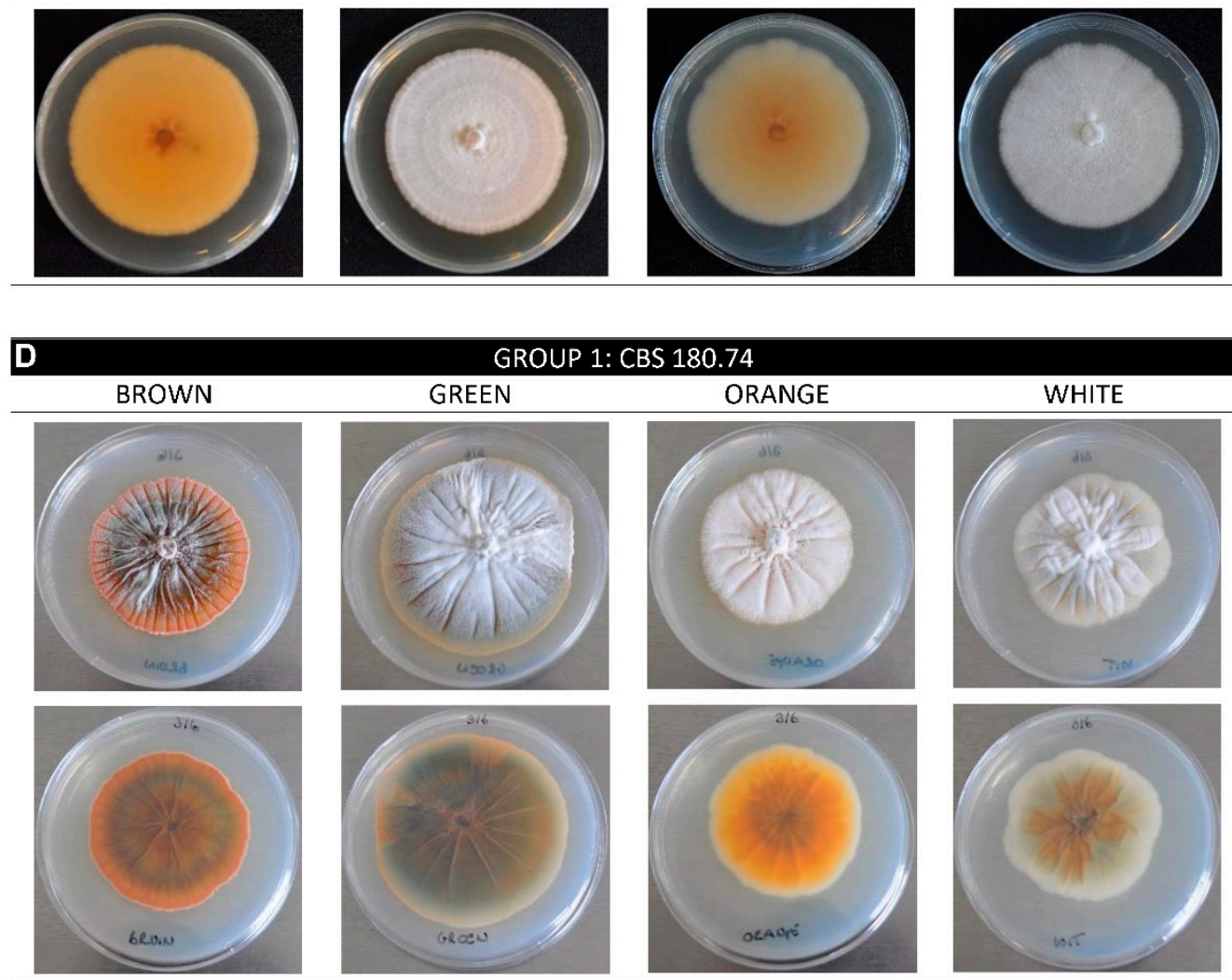

GROUP 1: CBS 180.74
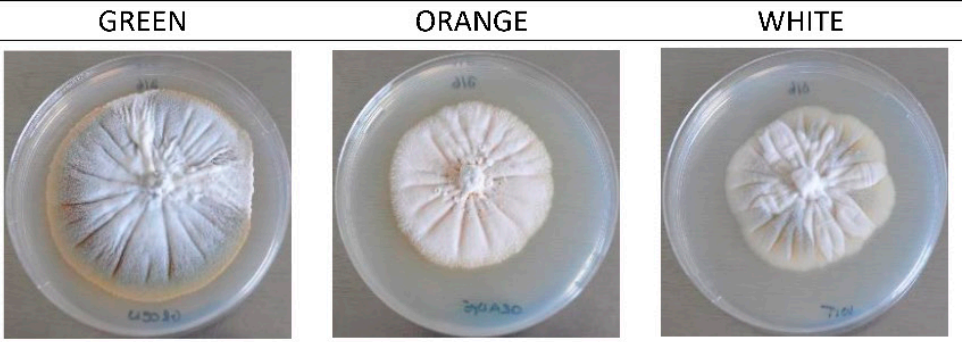

Figure 2. S. oryzae shown on PDA. (A-C) Isolates of Group 1, 2 and 3 when grown for 15 days in dark at $28^{\circ} \mathrm{C}$. Left is reverse view, right is front view. Sectorisation is clearly visible for IBNG0013 (Group 1) and RFNG33 (Group 2). (D) Isolated sectors of the Group 1 strain CBS180.74, grown for 21 days in dark at $28{ }^{\circ} \mathrm{C}$. Top is front view, bottom is reverse view.

\subsection{Pathogenicity}

To evaluate the pathogenicity of S. oryzae, five isolates representing each of the three phylogenic groups were used in pathogenicity experiments. Plants of the japonica cultivar Kitaake were inoculated with all fifteen isolates. The disease development was evaluated at 8 days post-inoculation (DPI) 
by measuring the lesion area. The variation in virulence among the isolates of one group is high. For example, CBS414.81 caused a mean lesion area of $30 \mathrm{~mm}^{2}$, while BDNG0025 caused a mean lesion area of $278 \mathrm{~mm}^{2}$ (Figure 3A). The mean lesion area on Kitaake caused by Group 2 was significantly higher than the mean area caused by Group 1. Overall, Group 3 showed significantly higher pathogenicity than Groups 1 and 2, but all S. oryzae isolates tested were able to induce sheath rot symptoms on Kitaake (Figure 3).
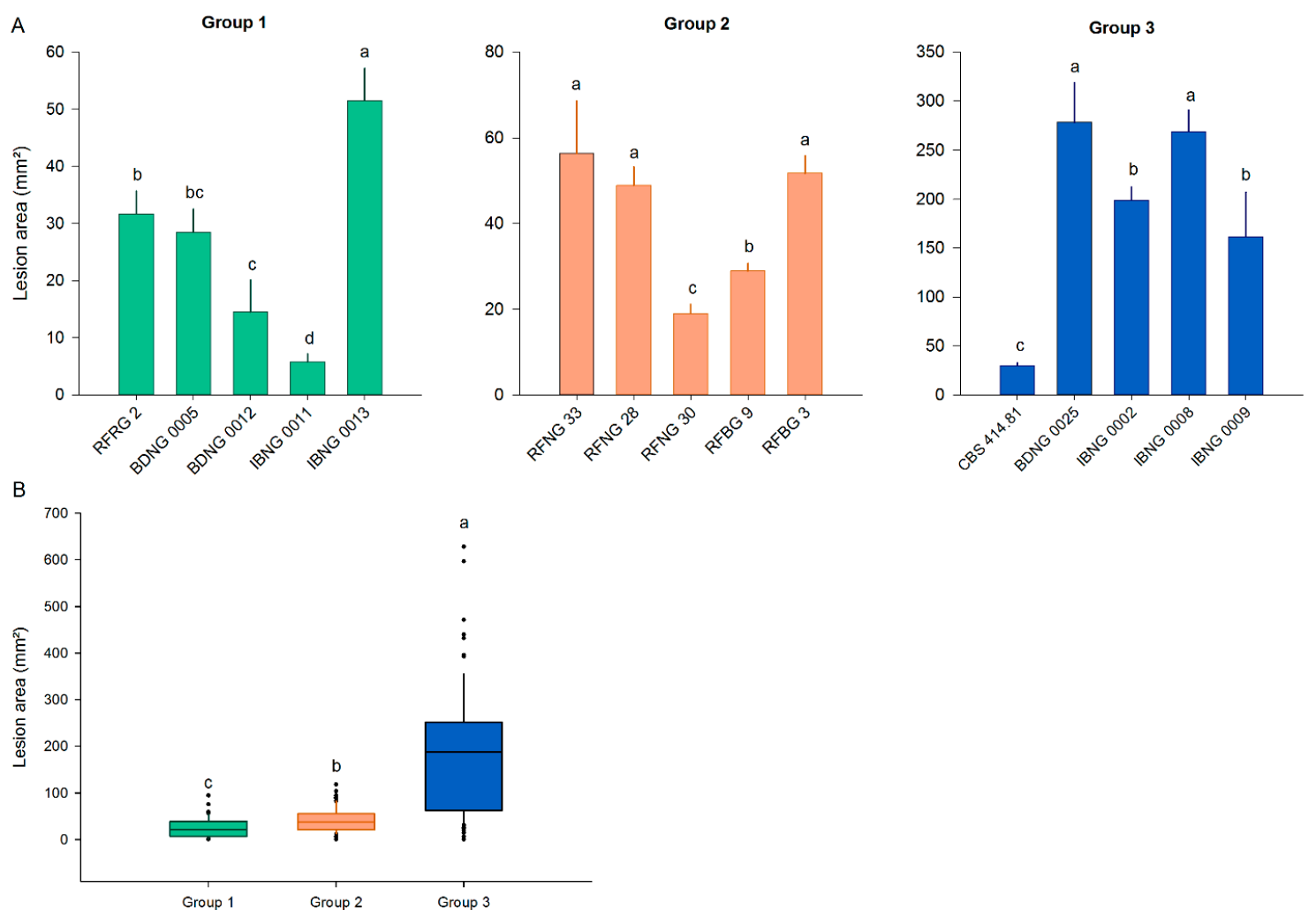

Figure 3. Pathogenicity data of $S$. oryzae isolates of three lineages on Kitaake rice plants. When 7 weeks old, rice plants were inoculated with S. oryzae isolates belonging to Groups 1,2 and 3 using the standard grain inoculum technique. At $8 \mathrm{DPI}$, the lesion area $\left(\mathrm{mm}^{2}\right)$ was measured. (A) Bars show the mean $\pm \mathrm{SE}$ of each isolate tested, with eighteen replicates for each isolate. Bars marked with different letters are statistically different (Mann-Whitney, $n=18, \alpha=0.05$ ). (B) Each boxplot shows the lesion area caused by all five isolates belonging to one lineage. Groups 1, 2 and 3 are represented by, respectively, green, orange and blue boxplots. All boxplots represent the median with the first and third quartile, the whiskers show the minimum and maximum values. Outliers and extreme values are represented by dots. Boxplots marked with different letters are statistically different (Mann-Whitney, $n=90, \alpha=0.05$ ).

\subsection{In Vitro Cerulenin and Helvolic Acid Production}

To evaluate the production of cerulenin and helvolic acid by S. oryzae, sixteen isolates belonging to the three phylogenic groups were used in in vitro experiments. For most Group 1 and 3 isolates, cerulenin levels were 10-20-fold higher than helvolic acid levels (Figure 4A,B). For Group 1 and 2 isolates, the in vitro production of cerulenin showed a very strong positive correlation with the in vitro production of helvolic acid (respectively, $\mathrm{r}=0.699, p<0.001, n=54$ and $\mathrm{r}=0.908, p<0.001, n=54$ ). A weak, negative, non-significant correlation was observed for the in vitro production of both toxins by isolates of Group $3\left(r_{\mathrm{s}}=-0.241, p=0.246, n=54\right)$. 

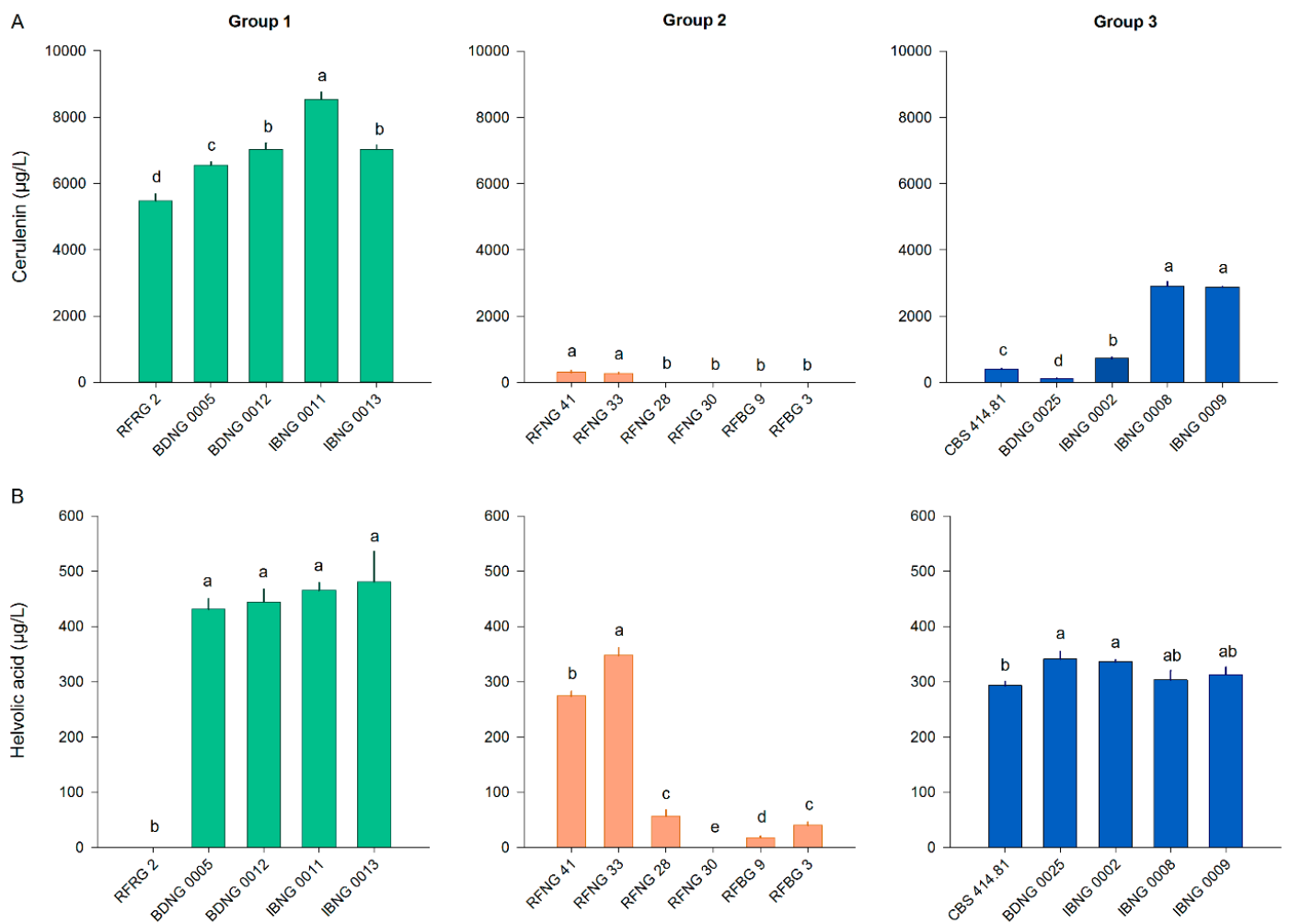

Figure 4. Cerulenin and helvolic acid production by S. oryzae isolates on PDA. S. oryzae isolates of Groups 1, 2 and 3 were grown for 7 days on PDA. The concentration of $(\mathbf{A})$ cerulenin $(\mu \mathrm{g} / \mathrm{L})$ and $(\mathbf{B})$ helvolic acid $(\mu \mathrm{g} / \mathrm{L})$ in the extract of the culture was measured with liquid chromatography high-resolution mass spectrometry. Isolates of Groups 1, 2 and 3 are represented by, respectively, green, orange and blue bars. All bars show the mean \pm SE of the toxin levels with five replicates for each isolate. Bars marked with different letters are statistically different (Mann-Whitney, $n=5, \alpha=0.05$ ).

With a mean cerulenin production of $6922 \mu \mathrm{g} \mathrm{L}-1$, Group 1 contained the highest producers. Group 2 contained the lowest producers (on average $97 \mu \mathrm{g} \mathrm{L}{ }^{-1}$ ) and isolates of Group 3 produced moderate levels of cerulenin (on average $1409 \mu \mathrm{g} \mathrm{L}{ }^{-1}$ ). Despite the clear difference in the mean cerulenin production of the three phylogenic groups, there was a large variation in production among the isolates of one group. Only two isolates of Group 2, RFNG41 and RFNG33, produced cerulenin while no cerulenin could be detected in the PDA cultures of the other four isolates. Also Group 3 contained isolates producing cerulenin from 117 (BDNG0025) up to $2914 \mu \mathrm{g} \mathrm{L}^{-1}$ (IBNG0008) (Figure 4A). Although there was also a lot of variation in the helvolic acid production of the $S$. oryzae isolates, the differences among the three groups were smaller than in the cerulenin production. Group 1 isolates were the highest producers of helvolic acid with concentrations around $450 \mu \mathrm{g} \mathrm{L}^{-1}$ except for the Rwandan isolate RFRG2, which produced no detectable levels of helvolic acid (Figure 4B). Helvolic acid levels of Group 2 varied from no detection to $348 \mu \mathrm{g} \mathrm{L}^{-1}$. All isolates of Group 3, on the other hand, produced about $300 \mu \mathrm{g} \mathrm{L}^{-1}$ of helvolic acid (Figure $4 \mathrm{~B}$ ).

\subsection{Cerulenin and Helvolic Acid Production by Different Phenotypes of CBS180.74}

The conservation of the toxin production after sectorisation of CBS180.74 was studied. Therefore, all four phenotypes were grown in a liquid culture of which the extract was analysed by liquid chromatography high-resolution mass spectrometry. The results showed that the production of both cerulenin and helvolic acid was affected by sectorisation. The parent culture (white) produced the highest levels of both toxins (Figure 5). The green phenotype was most affected in its cerulenin production, with a decrease of almost $50 \%$. Compared to the parent culture, all phenotypes showed 
a very strong decrease in their helvolic acid production with no detection of helvolic acid in the orange culture at all (Figure 5).

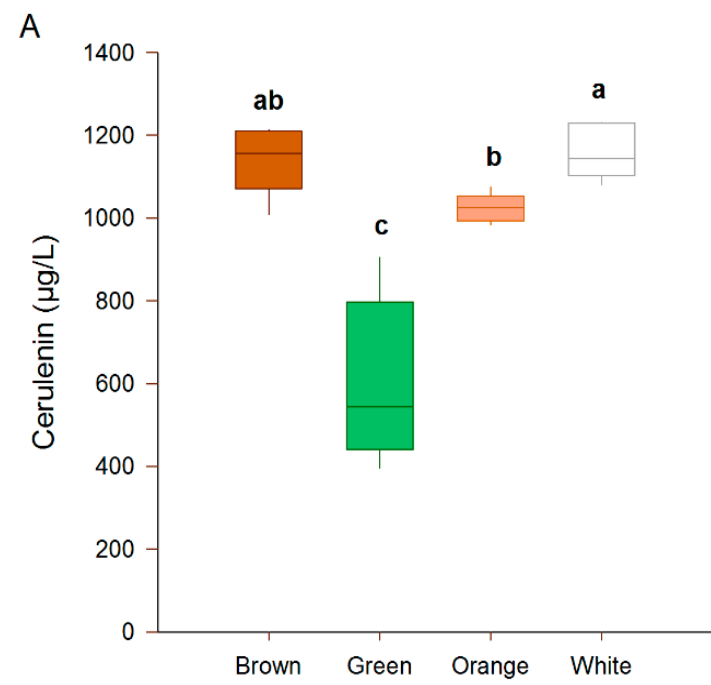

S. oryzae CBS180.74 phenotypes

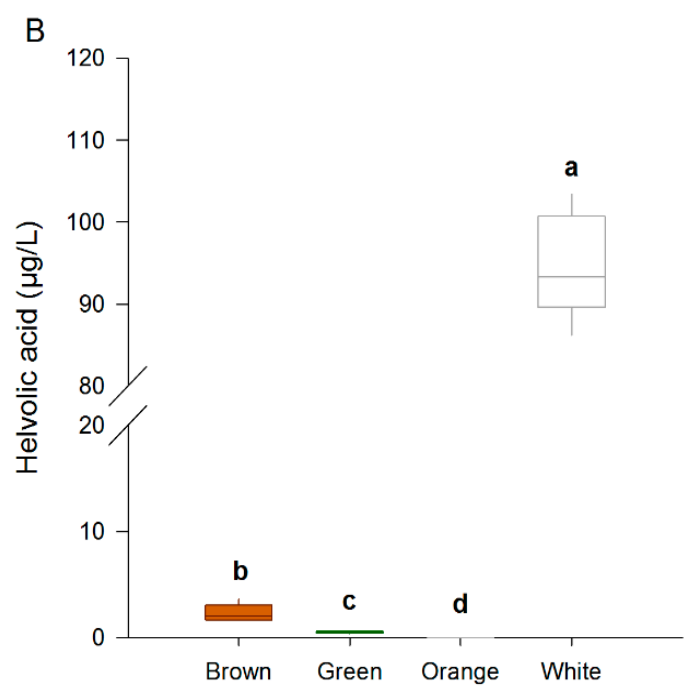

S. oryzae CBS180.74 phenotypes

Figure 5. The levels of (A) cerulenin and (B) helvolic acid produced in vitro by different phenotypes of the S. oryzae isolate CBS180.74. Four isolated phenotypes of the Group 1 isolate CBS180.74 were grown in a liquid culture for 7 days. All boxplots represent the median with the first and third quartile; the whiskers show the minimum and maximum values. Boxplots marked with different letters are statistically different (Mann-Whitney, $n=5, \alpha=0.05$ ).

\subsection{A Comparison of the Toxin Production in Vitro and in Planta}

Ten S. oryzae isolates representing the three phylogenic groups were used to compare toxin production in vitro and in the host plant. Starting from the same culture on PDA, in vitro cultures for toxin measurements and grain inoculum for rice inoculation were set up. Cerulenin and helvolic acid levels were measured in 7-day-old in vitro cultures and the rice sheath of plants at 6 DPI. At day 7, the high cerulenin-producing isolates IBNG0008, IBNG0009, RFRG2, CBS180.74 and BDNG0005 produced about ten times more cerulenin than helvolic acid on PDA (Figure 6A,B). In the rice sheath however, at 6 DPI, the measured concentration of helvolic acid was higher than the cerulenin concentration except for RFRG2, for which no helvolic acid production could be detected in any condition (Figure 6C,D).

The in vitro production of cerulenin did not associate with its production in the host (Figure 7F). Nevertheless, the high cerulenin producers, such as IBNG0008, IBNG0009, RFRG2, CBS180.74 and BDNG0005, produced in both conditions significantly more cerulenin than the medium producers, BDNG0025 and RFNG41. Additionally, for isolates that did not produce any cerulenin on PDA (RFNG122, RFNG30 and RFBG3), no or very low levels (on average $1 \mathrm{ng} / \mathrm{g}, \mathrm{RFNG122}$ ) of cerulenin could be detected in the rice sheath (Figure 6A,C).

The in vitro production of helvolic acid did not correlate with the production in planta although the high in vitro-producing isolates (IBNG0008, IBNG0009, BDNG0025, CBS180.74, RFNG41 and BDNG0005) produced high to moderate amounts in the rice plant (Figure 7E). The production profiles of the low producing isolates, RFNG122 and RFBG3, were similar in both conditions and RFRG2 produced no helvolic acid in culture, nor in the rice sheath (Figure 6A-D). 
A

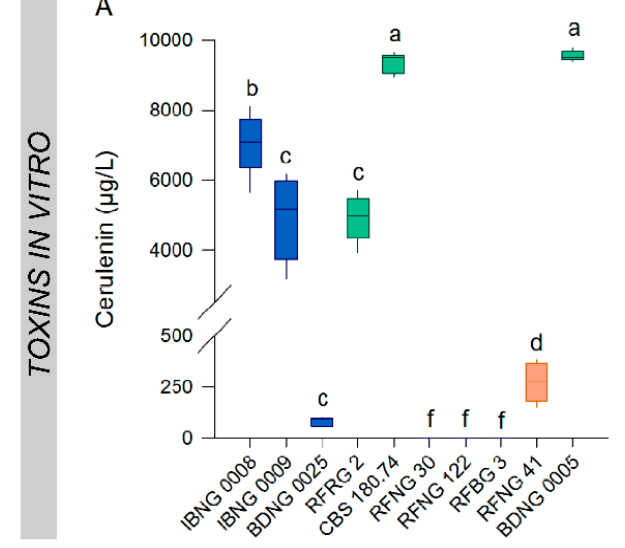

C

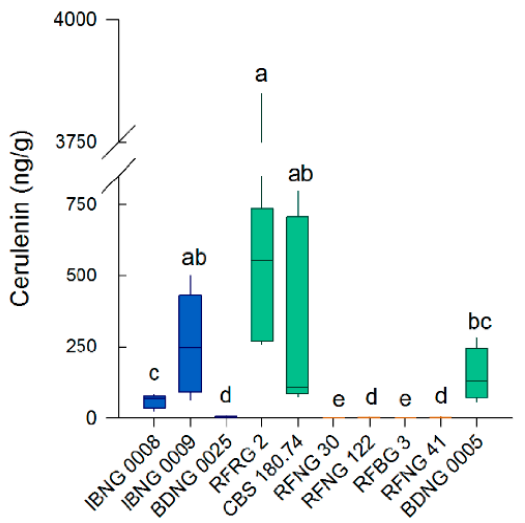

B

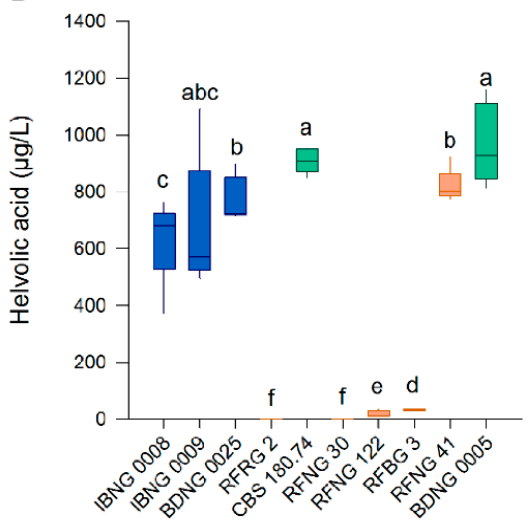

D

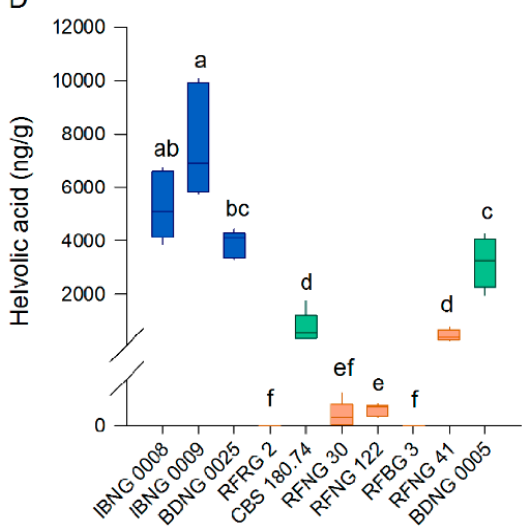

$\mathrm{E}$

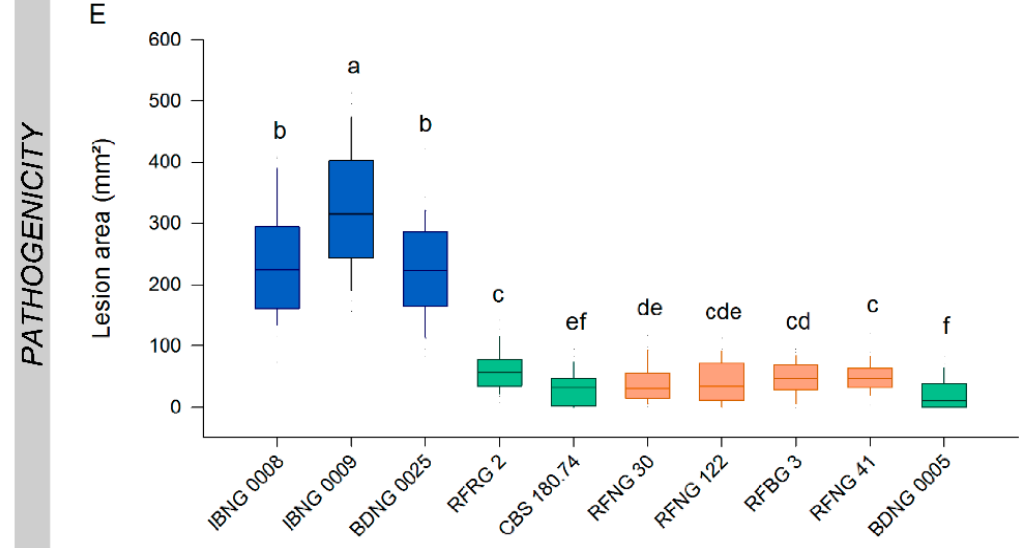

Figure 6. Toxin production in vitro and in planta versus pathogenicity on rice. S. oryzae isolates were grown for 7 days on PDA. The concentration of $(\mathbf{A})$ cerulenin $(\mu \mathrm{g} / \mathrm{L})$ and $(\mathbf{B})$ helvolic acid $(\mu \mathrm{g} / \mathrm{L})$ in the extract of the culture was measured with liquid chromatography high-resolution mass spectrometry. When 7 weeks old, rice plants were inoculated with the same strains of $S$. oryzae using the standard grain inoculum technique. At 6 DPI, (C) cerulenin (ng/g) and (D) helvolic acid (ng/g) levels were analysed in sheath samples and (E) the disease was scored by measuring the lesion area $\left(\mathrm{mm}^{2}\right)$. Isolates of Groups 1, 2 and 3 are represented by, respectively, green, orange and blue boxplots. All boxplots represent the median with the first and third quartile, the whiskers show the minimum and maximum values. Outliers and extreme values are represented by dots. The boxplots show data of 1 experiment with five replicates for each isolate for toxin concentrations (A-D) and 25 replicates for each isolate for the lesion area $(\mathbf{E})$. Boxplots marked with different letters are statistically different (Mann-Whitney, $n=5$ or $n=25, \alpha=0.05$ ). 
A

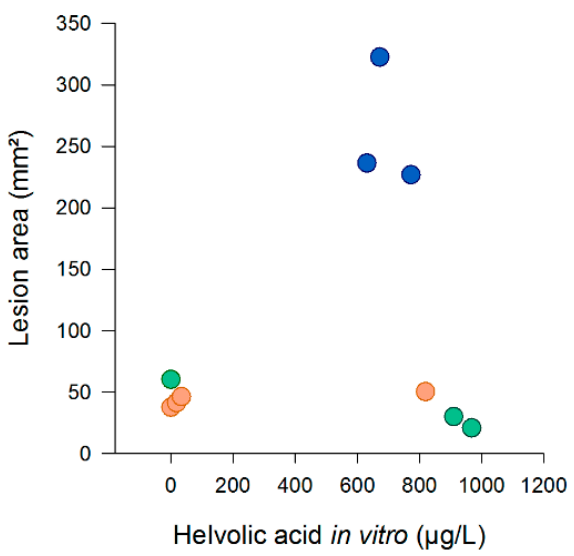

C

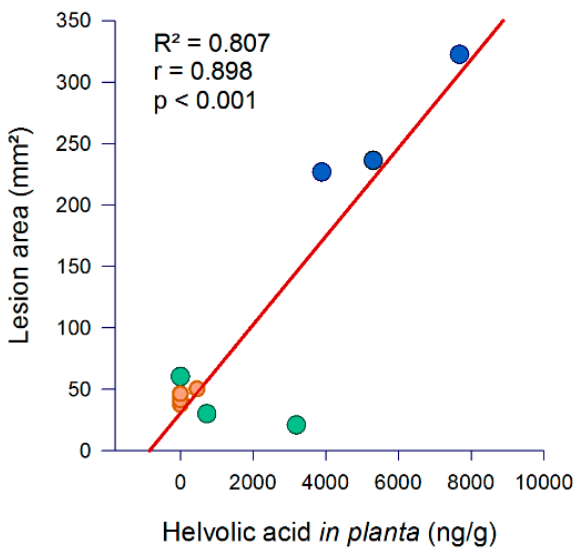

E

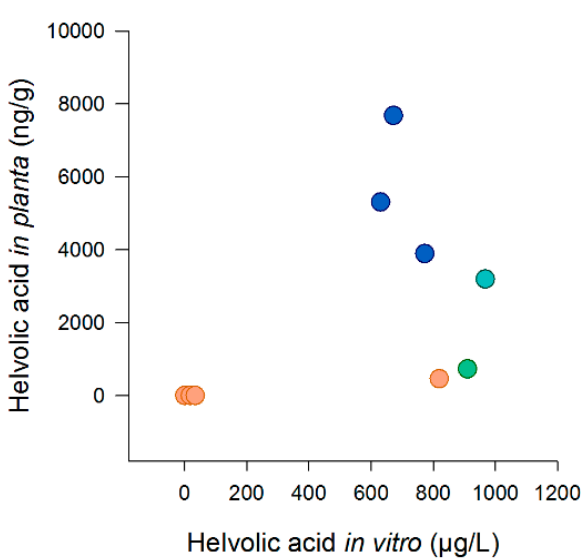

B

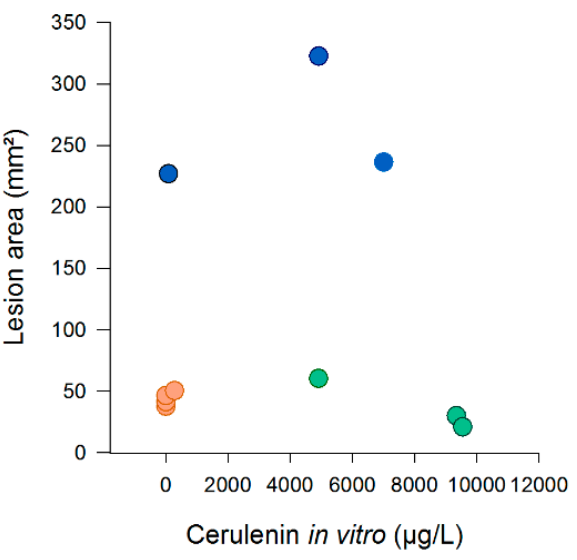

D

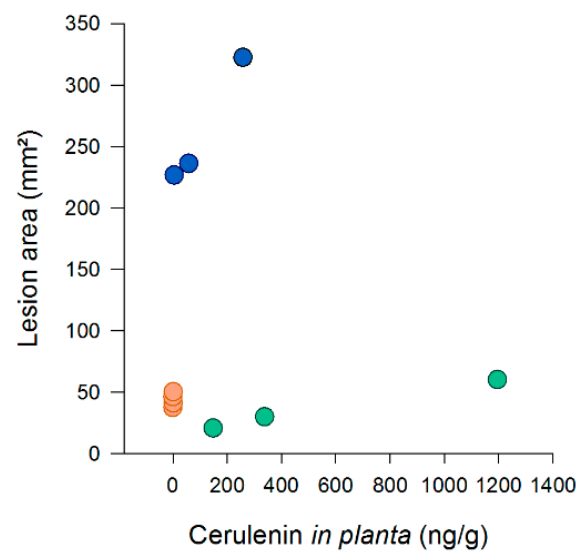

F

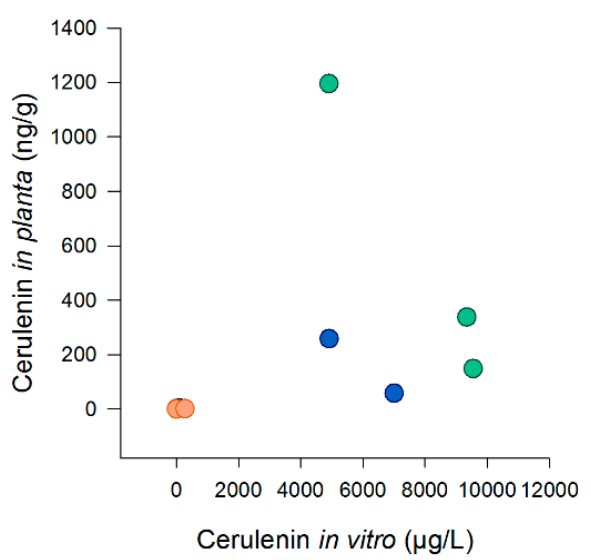

Figure 7. Associations between toxin production in vitro, in planta and pathogenicity on rice. S. oryzae isolates were grown for 7 days on PDA. The concentration of cerulenin $(\mu \mathrm{g} / \mathrm{L})$ and helvolic acid $(\mu \mathrm{g} / \mathrm{L})$ in the extract of the culture was measured with liquid chromatography high-resolution mass spectrometry. When 7 weeks old, rice plants were inoculated with the same strains of S. oryzae using the standard grain inoculum technique. At 6 DPI, cerulenin (ng/g) and helvolic acid (ng/g) levels were analysed in sheath samples and the disease was scored by measuring the lesion area $\left(\mathrm{mm}^{2}\right)$. Scatterplots show the associations between pathogenicity and helvolic acid and cerulenin levels in vitro (A, B) and in planta $(\mathbf{C}, \mathbf{D})$ and between the production in vitro and in planta of helvolic acid (E) and cerulenin (F). Isolates of Groups 1, 2 and 3 are represented by, respectively, green, orange and blue dots; significant linear correlations are represented by the linear regression curve in red. 


\subsection{Disease Severity Correlates with In Planta Helvolic Acid Production}

In order to correlate toxin levels with the severity of the caused symptoms, disease development was evaluated by measuring the lesion area (Figure 6E). In vitro production of cerulenin or helvolic acid was not correlated with pathogenicity (Figure 7A,B). Cerulenin levels in the rice sheath did not associate with the lesion area either (Figure 7D). The production of helvolic acid in the rice sheath, however, showed a very strong, positive, linear correlation with the disease severity $(\mathrm{r}=0.898, p<$ $0.001, n=10$; Figure 7C).

\section{Discussion}

In this study, S. oryzae isolates from an international collection were used to describe the morphological, toxigenic and pathogenic variations. The population of $S$. oryzae can be divided into three distinct lineages [31]. Concerning the high level of conservation of housekeeping genes, this distinction suggests that the population of $S$. oryzae is very diverse. This observation was confirmed by the phenotypic and pathogenic characterisation of the isolates used in this study. Especially when grown on PDA, the isolates belonging to Groups 1 and 2 showed a variable growth rate and colour pattern. Isolates from Group 3 were more uniform in growth rate and colour than isolates from Groups 1 and 2. In addition, isolates from Groups 1 and 2 were less pathogenic than isolates belonging to Group 3.

To investigate these differences in pathogenicity between the lineages and among the isolates of the groups, the production of the phytotoxins cerulenin and helvolic acid in PDA cultures was compared with the production in host tissue. Cerulenin production was highest for Group 1 isolates and Group 2 isolates produced no or very low levels of cerulenin in vitro. Helvolic acid production, on the other hand, was not correlated with the phylogenetic groups. The observed levels of both toxins in culture were in agreement with the levels measured by Ayyadurai et al. (2005) [12]. Up till now, these toxins were thought to be the main pathogenicity factors of $S$. oryzae [34]. Isolates that produce high amounts of helvolic acid and/or cerulenin in culture were described to cause a higher disease incidence $[12,14]$. In this work, however, these observations could not be confirmed since the production of cerulenin or helvolic acid by an isolate in vitro did not correlate with its pathogenicity.

To the best of our knowledge, neither cerulenin nor helvolic acid has previously been measured in the plant. In this work, a new LC-MS/MS method was established to reliably quantify both toxins in the rice sheath. Interestingly, the production of cerulenin and helvolic acid in vitro did not correlate with the production in the rice sheath. Nor did the cerulenin levels in the rice sheath associate with pathogenicity. A strong positive correlation, however, was observed between pathogenicity and in planta helvolic acid levels, with the highest in planta helvolic acid producers all belonging to Group 3. The fact that Group 3 isolates are able to produce high levels of helvolic acid in the rice sheath, and therefore are more virulent than the isolates of the other two groups, could possibly be attributed to a lack of sectorisation. Isolates of Group 3 were phenotypically stable in culture, while most isolates of Groups 1 and 2 formed morphological variants, called sectors. Sectorisation can be the result of genetic changes, such as spontaneous mutations or transposons, of cultural degeneration caused by ageing or stress conditions, epigenetic changes or mycovirus infection [23,26,28,35]. Sectors are often impaired in their ability to produce secondary metabolites $[23,36]$. This was confirmed in this study for the Group 1 isolate CBS180.74. Helvolic acid levels were much lower in the liquid cultures of all morphological variants compared to the parent culture. Also, the cerulenin production by the green and orange variants was lower than the production by the parent culture. Due to this decrease in toxin production, unstable isolates are often less virulent [23,26,35].

Shah and Butt (2005) [26] describe that more sectors are formed in nutrient-rich medium. As the growth of $S$. oryzae isolates was more stable on OA medium than on PDA, our study confirms these observations. In PDA, high nutrient levels could cause osmotic stress, leading to sectorisation [37]. In planta, S. oryzae encounters multiple stresses, including oxidative stress [35]. During the invasion of the host, a pathogen is subjected to high levels of reactive oxygen species (ROS) produced by both 
the pathogen itself and by the host, which leads to phenotypic degradation [38]. The lack of sector formation by isolates of Group 3 could possibly indicate that this lineage is more tolerant to stress.

Another explanation for the observed differences in stability and virulence between Group 3 and the Group 1 and 2 isolates could be a viral infection of the latter. Mycovirus infection is a well-described cause of sectorisation in plant pathogens, leading to hypovirulence $[25,36]$. The spread of these mycoviruses occurs mainly through hyphal anastomosis. This process of horizontal gene transmission occurs between vegetative compatible isolates [24,36,39]. Since we only observe sectorisation and hypovirulence in the isolates of two groups (Groups 1 and 2), vegetative incompatibility with Group 3 could possibly explain the high virulence and stability of the Group 3 isolates [31,32]. The difference in toxin production between the in vitro conditions and the rice sheath could also indicate that the isolates of Groups 1 and 2 are indeed infected with a mycovirus [39]. Brusini and Robin (2013) [40] observed that the transmission rate of mycoviruses is higher in the host plant of the pathogenic fungus, leading to more symptoms of the viral infection in planta than in vitro. Both phenotypic degradation and mycovirus infection can lead to a decreased production of the phytotoxins, resulting in lower pathogenicity. In accordance with this hypothesis, we observed a decrease in the relative toxin production of the isolates of Groups 1 and 2 in the host tissue compared to the production on PDA.

Based on these results, we conclude that in planta toxin measurements are more relevant to investigate the role of the phytotoxins in the etiology of sheath rot. As the levels of helvolic acid in infected tissue strongly correlate with the pathogenicity, our data suggest that helvolic acid is an important pathogenicity factor of $S$. oryzae. We also conclude that not only the competence to produce helvolic acid is an important trait, but also the ability to maintain this production in stressed conditions. S. oryzae is also known for its production of cell wall degrading enzymes [12]. As the production of pathogenicity-related enzymes may be affected during sectorisation, a decrease in this enzyme activity could possibly explain the low levels of pathogenicity of the high helvolic acid-producing isolate BDNG0005, which clusters in Group 1 [26].

To further investigate this hypothesis, future research should study the presence and transmission of mycoviruses in the different phylogenetic groups of S. oryzae. Also, stress resistance and its relation to pathogenicity and toxin production of the different groups should be studied.

\section{Materials and Methods}

\subsection{Fungal Isolates}

In this study, isolates from traditional rice-growing areas in Rwanda (Bugarama, Nyagatare and Rwagitima, and Rugeramigozi) and Nigeria (Ibadan and Badaggi) were used together with isolates from the CBS collection (Table A1) [31,32,41]. Pure cultures were stored on PDA (Difco) slants and at $-80{ }^{\circ} \mathrm{C}$ in $20 \%$ glycerol.

\subsection{Morphological Characterization}

To measure growth, isolates were grown on $50 \%$ OA (Difco) and PDA plates at $28^{\circ} \mathrm{C}$. During 15 days, colony diameters were measured every 3 days. For each plate, two perpendicular diameters were measured, of which the average was used for the radial growth rate calculations. The growth rate was calculated by performing a linear regression on the average diameter at five different time points. For each isolate, the radial growth rate was measured three times on each medium.

\subsection{Pathogenicity Assays}

Fifteen isolates representing all three groups were selected for pathogenicity tests on rice plants. Isolates from Rwanda (regions Bugarama, Nyagatare and Rwagitima, and Rugeramigozi), Nigeria (regions Ibadan and Badaggi) and the CBS collection were included in the pathogenicity test. 
Inoculum was prepared according to the standard grain inoculum technique [42]. Briefly, rice grains were soaked in water for $60 \mathrm{~min}$, excess water was removed, and the grains were autoclaved twice on two different days.

For each $4 \mathrm{~g}$ of rice grains, one plug (diameter $=5 \mathrm{~mm}$ ) from the edge of a 14-day-old fungal colony was added together with $1 \mathrm{~mL}$ of sterile distilled water. Every 2 days, the grain inoculum was shaken to prevent the formation of clumps. After 14 days of incubation at $28^{\circ} \mathrm{C}$, the inoculum was fully colonised.

Rice seeds of the japonica cultivar Kitaake were dehulled and surface sterilised in $2 \%$ sodium hypochlorite solution for $25 \mathrm{~min}$, rinsed five times in sterile distilled water, and placed in Petri dishes containing sterile moistened filter papers (Whatman, grade 3 ). After 1 week of incubation at $28^{\circ} \mathrm{C}$, seedlings were sown in perforated plastic trays $(22 \times 15 \times 6 \mathrm{~cm})$ containing sterile potting soil (Structural; Snebbout, Kaprijke, Belgium). Rice seedlings were maintained in a greenhouse $\left(28^{\circ} \mathrm{C}, 60 \%\right.$ relative humidity) and fertilized weekly with $0.2 \%$ iron sulphate and $0.1 \%$ ammonium sulphate.

One fully colonised grain was introduced in the junction point between the sheath of the second youngest plant leaf and the stem. Inoculation points were covered with moist cotton and wrapped with parafilm to maintain humidity. Plants were incubated under growth chamber conditions $\left(28^{\circ} \mathrm{C}\right.$ day $/ 28{ }^{\circ} \mathrm{C}$ night, $12 / 12$ light regimen, and $85 \%$ relative humidity during the first $24 \mathrm{hrs}, 65 \%$ relative humidity during day 2-8). The disease development was evaluated 8 days after inoculation by measuring the lesion area $(\pi \times($ lesion length $/ 2) \times($ lesion width/2) $)$ on the flag leaf sheath. For each taxonomic group, the experiments included five isolates with distinct morphologic characteristics. Each treatment consisted of three trays containing five plants.

\subsection{Chemical Analysis}

\subsubsection{Extraction from PDA}

The toxin production in solid medium was measured for all fifteen isolates used in the pathogenicity experiments and the Group 2 isolate, RFNG41. Each isolate was grown in one 6-well plate with each well containing $3 \mathrm{~mL}$ of PDA. Five wells were inoculated with one plug of a 2-week-old fungal colony, and one non-inoculated well was used as a control for contamination. After a seven-day incubation period at $28^{\circ} \mathrm{C}$ in constant dark, the total content of each well was cut in small pieces and toxins were extracted in an hour with $3 \mathrm{~mL}$ of chloroform [12,43]. Agar and mycelium were removed by filtration using sterile filter paper (Whatmann, grade 3 ). The collected chloroform phase was reduced to dryness under $\mathrm{N}_{2}$ at $20{ }^{\circ} \mathrm{C}$ in a Turbovap ${ }^{\circledR} \mathrm{LV}$ automated concentration evaporator (Biotage, Uppsala, Sweden). After reconstituting the extract with methanol/water (20:80 $v / v)$ containing $0.1 \%$ formic acid, samples were ready for instrumental analysis.

\subsubsection{Extraction from Liquid Culture}

For the extraction from a liquid culture, S. oryzae isolates were grown for 7 days at $28^{\circ} \mathrm{C}$ in total dark in still liquid culture in test tubes. Every isolate was grown in five test tubes, each containing $10 \mathrm{~mL}$ liquid medium and three plugs (diameter $5 \mathrm{~mm}$ ) of a 2-week-old fungal colony grown on PDA. The liquid medium contained $1 \%$ glucose, $3 \%$ glycerol, $0.5 \%$ peptone and $0.2 \% \mathrm{NaCl}$ [44]. The 7 -day-old liquid culture was filtered using sterile filter paper (Whatman, grade 3) to remove the mycelium after which the filtrate was filter-sterilized with a $0.45 \mu \mathrm{m}$ syringe filter unit (Millex). An equal amount of chloroform was added to the sterile filtrate and after shaking for $10 \mathrm{~s}$, the filtrate was incubated for $1 \mathrm{~h}$ in the dark [12]. After incubation, the water phase was collected and discarded, leaving a pure chloroform extract. Further steps are described in Section 4.4.1.

\subsubsection{Extraction from Plant Material}

The plants were inoculated with ten $S$. oryzae isolates belonging to the three phylogenic groups using the standard grain inoculum technique as described above. At 6 DPI, samples were collected by 
pooling the inoculated area of the sheath of all five plants of one tray. The samples were immediately immersed into liquid $\mathrm{N}_{2}$ and stored at $-80^{\circ} \mathrm{C}$. Just before the extraction, the material was ground using a tissuelyser. Of this plant powder, $100 \mathrm{mg}$ was extracted by adding $5 \mathrm{~mL}$ cold (approximately $4{ }^{\circ} \mathrm{C}$ ) modified Bieleski solvent (methanol, ultrapure water and formic acid, 75:20:5) and vortexing for $10 \mathrm{sec}$. After $20-24 \mathrm{hrs}$ of incubation at $-80^{\circ} \mathrm{C}, 4 \mathrm{~mL}$ of the supernatant was filtered ( $30 \mathrm{kDa}$ Amicon Ultra centrifugal filter, Merck Millipore, Overijse, Belgium) for $30 \mathrm{~min}$ at 3,900 rpm at $4{ }^{\circ} \mathrm{C}$ in a SW9 R centrifuge (Froilabo, Paris, France). Next, $2.5 \mathrm{~mL}$ of the filtrate was reduced to dryness under $\mathrm{N}_{2}$ at $20{ }^{\circ} \mathrm{C}$ using a Turbovap ${ }^{\circledR}$ evaporator. When the extract was reconstituted with $0.5 \mathrm{~mL}$ methanol/water $(20: 80 \mathrm{v} / \mathrm{v})$ with $0.1 \%$ formic acid, it was ready for instrumental analysis.

\subsubsection{Instrumental Analysis}

Instrumental analysis was performed on an ultra-high performance liquid chromatography system coupled to a quadrupole-orbitrap mass spectrometer, as described in detail by Haeck et al. (2018) [45]. Chromatographic separation was achieved on an Accela U-HPLC pumping system (Thermo Fisher Scientific, Erembodegem, Belgium), coupled to an Accela autosampler and degasser and equipped with a Nucleodur C18 column ( $50 \times 2 \mathrm{~mm} ; 1.8 \mu \mathrm{m} \mathrm{d}_{\mathrm{p}}$, Macherey-Nagel, Düren, Germany). The mobile phase consisted of (A) methanol with $0.01 \%$ formic acid and (B) water with $0.1 \%$ formic acid, and the linear gradient was (for solvent A): $0-1 \mathrm{~min}$ at 20\%, 1-2.5 $\mathrm{min}$ from $20-45 \% ; 2.5-9 \mathrm{~min}$ from $45-100 \%$; $9-10 \mathrm{~min}$ at $100 \% ; 10-14 \mathrm{~min}$ at $20 \%$. Mass spectrometric analysis was carried out using a Q-Exactive ${ }^{\mathrm{TM}}$ bench top HRMS (Thermo Fisher Scientific Erembodegem, Belgium), equipped with a heated electrospray ionization source operating in the positive ionisation mode. The measurements were performed in targeted single ion monitoring (t-SIM) at a mass resolving power of 70,000 full width at half maximum (FWHM). The method has been validated according to Haeck et al. (2018) [45] by the use of analytical standards ( $>96 \%$ purity) purchased at Abcam (Cambridge, UK). The analytical process efficiency, including both the extraction recovery and the matrix effects, ranged between $70 \%$ (helvolic acid) and $114 \%$ (cerulenin). Interday repeatability data showed good precision, with relative standard deviations (RSD, $n=3$ ) lower than $10 \%$ at concentration levels close to the analyte's limits of detection $(0.05$ and $0.08 \mu \mathrm{g} . \mathrm{L}^{-1}$ for helvolic acid and cerulenin, respectively). The linearity of the instrumental analysis is expressed by a $\mathrm{R}^{2}>0.965(n=8)$ within at least three orders of magnitude.

\subsection{Statistical Analysis}

All statistical tests were performed with SPSS 25.0 (IBMSPSS, Armonk, NY, USA) with a significance level fixed at 0.05 . To test the assumption of normality, the Shapiro-Wilk test (Bonferroni correction) was used. Homoscedasticity was tested using Levene's test. If both assumptions were fulfilled, a one-way ANOVA (post-hoc: Tukey-Bonferroni) test was used to compare the means. In case at least one of these assumptions was not met, the non-parametric Kruskal-Wallis rank sum test was performed, followed by the Mann-Whitney test for a pairwise comparison. To test for correlations, the Pearson correlation $(r)$ was used. If the relationship was non-linear, Spearman's rank $\left(\mathrm{r}_{\mathrm{s}}\right)$ correlation was applied.

Author Contributions: Conceptualization of this study was made by M.H., K.A. and K.J.P.; methodology was optimized by K.J.P., A.H. and K.D., investigation and data collection was performed by K.J.P., L.H. and O.O.A., formal analysis and visualization by K.J.P., writing—original draft preparation by K.J.P. and A.H.; writing-review and editing, K.J.P., K.D., K.A. and M.H.; funding acquisition and project administration by M.H., K.A., K.D. and overall supervision by M.H. All authors have read and agreed to the published version of the manuscript.

Funding: This research was funded by the Fund for Scientific Research-Flanders (FWO), grant number G031317N. Also the Special Research Fund of Ghent University is acknowledged for its financial support in the GOA 01GB3013 project. The authors acknowledge the financial support (AUGE/11/016) from the Hercules Foundation of the Flemish Government for the UHPLC-Q-ExactiveTM MS equipment used for chemical analysis.

Acknowledgments: We want to thank the lab technician Nadia Lemeire for the help during rice inoculations. Also special thanks to the greenhouse responsible Stefan Vidts for taking care of the rice plants. Illustrations in the graphical abstract were partially created with BioRender.com. 
Conflicts of Interest: The authors declare no conflict of interest. The funders had no role in design, execution, interpretation or writing of this study.

\section{Appendix A}

Table A1. Overview of the phylogeny and origin of the S. oryzae isolates used in this study.

\begin{tabular}{|c|c|c|c|}
\hline Isolate & Origin & Phylogenic Group & Reference \\
\hline CBS 180.74 & India & 1 & [33] \\
\hline CBS 361.75 & Kenya & 1 & [31] \\
\hline CBS 120.817 & Panama & 1 & [31] \\
\hline CBS 485.80 & Australia & 1 & [31] \\
\hline RFRG 2 & Rwanda, Rugeramigozi & 1 & [31] \\
\hline BDNG 0004 & Nigeria, Badaggi & 1 & [31] \\
\hline BDNG 0005 & Nigeria, Badaggi & 1 & [31] \\
\hline BDNG 0007 & Nigeria, Badaggi & 1 & [31] \\
\hline BDNG 0009 & Nigeria, Badaggi & 1 & [31] \\
\hline BDNG 0012 & Nigeria, Badaggi & 1 & [31] \\
\hline BDNG 0014 & Nigeria, Badaggi & 1 & [31] \\
\hline BDNG 0022 & Nigeria, Badaggi & 1 & [31] \\
\hline BDNG 0023 & Nigeria, Badaggi & 1 & [31] \\
\hline IBNG 0011 & Nigeria, Ibadan & 1 & [31] \\
\hline IBNG 0012 & Nigeria, Ibadan & 1 & [31] \\
\hline IBNG 0013 & Nigeria, Ibadan & 1 & [31] \\
\hline CBS 399.73 & India & 2 & [33] \\
\hline CBS 101.61 & Mexico & 2 & [31] \\
\hline RFNG 41 & Rwanda, Nyagatare and Rwagitama & 2 & [31] \\
\hline RFNG 33 & Rwanda, Nyagatare and Rwagitama & 2 & [31] \\
\hline RFNG 30 & Rwanda, Nyagatare and Rwagitama & 2 & [31] \\
\hline RFNG 28 & Rwanda, Nyagatare and Rwagitama & 2 & [31] \\
\hline RFNG 122 & Rwanda, Nyagatare and Rwagitama & 2 & [31] \\
\hline RFBG 3 & Rwanda, Bugarama & 2 & [31] \\
\hline RFBG 9 & Rwanda, Bugarama & 2 & [31] \\
\hline CBS 414.81 & Nigeria & 3 & [33] \\
\hline BDNG 0025 & Nigeria, Badaggi & 3 & [31] \\
\hline IBNG 0002 & Nigeria, Ibadan & 3 & [31] \\
\hline IBNG 0008 & Nigeria, Ibadan & 3 & [31] \\
\hline IBNG 0009 & Nigeria, Ibadan & 3 & [31] \\
\hline IBNG 0001 & Nigeria, Ibadan & 3 & [31] \\
\hline
\end{tabular}

\section{References}

1. Mohanty, S. Trends in global rice consumption. Rice Today 2013, 12, 44-45.

2. Fageria, N. Yield physiology of rice. J. Plant Nutr. 2007, 30, 843-879. [CrossRef]

3. FAO Rice Market Monitor (RMM); Food and Agriculture Organization United Nation: Rome, Italy, 2018.

4. Saito, K.; Dieng, I.; Toure, A.; Somado, E.; Wopereis, M. Rice yield growth analysis for 24 African countries over 1960-2012. Glob. Food Sec. 2015, 5, 62-69. [CrossRef]

5. Demont, M. Reversing urban bias in African rice markets: A review of 19 national rice development strategies. Glob. Food Sec. 2013, 2, 172-181. [CrossRef]

6. Balamurugan, P.; Balasubramanian, V. Challenges and opportunities for increasing rice production in sub saharan Africa. J. Innov. Agric. 2017, 4, 1-10.

7. Sakthivel, N. Sheath rot disease of rice: Current status and control strategies. In Major Fungal Diseases of Rice: Recent Advances; Sreenivasaprasad, S., Johnson, R., Eds.; Kluwer Academic Publishes: Puducherry, India, 2001; pp. 271-283.

8. Mvuyekure, S.; Sibiya, J.; Derera, J.; Nzungize, J.; Nkima, G. Genetic analysis of mechanisms associated with inheritance of resistance to sheath rot of rice. Plant Breed. 2017, 136, 509-515. [CrossRef] 
9. Mvuyekure, S.; Sibiya, J.; Derera, J.; Nzungize, J.; Nkima, G. Assessment of genetic diversity of rice based on SNP markers for selection of parents for sheath rot (Sarocladium oryzae) resistance breeding. S. Afr. J. Plant Soil 2018, 35, 51-59. [CrossRef]

10. Bigirimana, V.; Hua, G.; Nyamangyoku, O.; Höfte, M. Rice Sheath Rot: An emerging ubiquitous destructive disease complex. Front. Plant Sci. 2015, 6, 1066. [CrossRef]

11. Nandakumar, R.; Babu, S.; Viswanathan, R.; Sheela, J.; Raguchander, T.; Samiyappan, R. A new bio-formulation containing plant growth promoting rhizobacterial mixture for the management of sheath blight and enhanced grain yield in rice. Biocontrol 2001, 46, 493-510. [CrossRef]

12. Ayyadurai, N.; Kirubakaran, S.; Srisha, S.; Sakthivel, N. Biological and molecular variability of Sarocladium oryzae, the sheath rot pathogen of rice (Oryza sativa L.). Curr. Microbiol. 2005, 50, 319-323. [CrossRef]

13. Tschen, J.; Chen, L.; Hsieh, S.; Wu, T. Isolation and phytotoxic effects of helvolic acid from plant pathogenic fungus Sarocladium oryzae. Bot. Bull. Acad. Sin. 1997, 38, 251-256.

14. Sakthivel, N.; Amudha, R.; Muthukrishnan, S. Production of phytotoxic metabolites by Sarocladium oryzae. Mycol. Res. 2002, 106, 609-614. [CrossRef]

15. Hoagland, R. Phytotoxicity of microbial antibiotics helvolic and fusidic acids. Allelopath. J. 2009, 23, 167-174.

16. Ratnaweera, P.; Williams, D.; de Silva, E.; Wijesundera, R.; Dalisay, D.; Andersen, R. Helvolic acid, an antibacterial nortriterpenoid from a fungal endophyte, Xylaria sp. of orchid Anoectochilus setaceus endemic to Sri Lanka. Mycology 2014, 5, 23-28. [CrossRef]

17. Hittalmani, S.; Mahesh, H.; Mahadevaiah, C.; Prasannakumar, M. De novo genome assembly and annotation of rice sheath rot fungus Sarocladium oryzae reveals genes involved in Helvolic acid and Cerulenin biosynthesis pathways. BMC Genomics 2016, 17, 271. [CrossRef]

18. Wenzel, M.; Patra, M.; Albrecht, D.; Chen, D.; Nicolaou, K.; Metzler-Nolte, N.; Bandow, J. Proteomic signature of fatty acid biosynthesis inhibition available for in vivo mechanism-of-action studies. Antimicrob. Agents Chemother. 2011, 55, 2590-2596. [CrossRef]

19. Gnanamanickam, S.; Mew, T. Interactions between Sarocladium oryzae and stem attacking fungal pathogens of rice. Plant. Soil 1991, 138, 213-219. [CrossRef]

20. Côrtes, M.; Silva-Lobo, V.; Filippi, M.; Lima, D.; Prabhu, A. Potential for using crude extract of Sarocladium oryzae for suppression of rice blast. Trop. Plant. Pathol. 2014, 39, 28-34. [CrossRef]

21. Brakhage, A. Regulation of fungal secondary metabolism. Nat. Rev. Microbiol. 2013, 11, 21-32. [CrossRef]

22. Fox, E.; Howlett, B. Secondary metabolism: Regulation and role in fungal biology. Curr. Opin. Microbiol. 2008, 11, 481-487. [CrossRef]

23. Ryan, M.; Bridge, P.; Smith, D.; Jeffries, P. Phenotypic degeneration occurs during sector formation in Metarhizium anisopliae. J. Appl. Microbiol. 2002, 93, 163-168. [CrossRef] [PubMed]

24. Kamaruzzaman, M.; He, G.; Wu, M.; Zhang, J.; Yang, L.; Chen, W.; Li, G. A novel partitivirus in the hypovirulent isolate qt5-19 of the plant pathogenic fungus Botrytis cinerea. Viruses 2019, 11, 24. [CrossRef] [PubMed]

25. Santos, V.; Mascarin, G.M.; da Silva Lopes, M.; Alves, M.C.D.F.; Rezende, J.M.; Gatti, M.S.V.; Dunlap, C.A.; Delalibera Júnior, Í. Identification of double-stranded RNA viruses in Brazilian strains of Metarhizium anisopliae and their effects on fungal biology and virulence. Plant. Gene 2017, 11, 49-58. [CrossRef]

26. Shah, F.; Butt, T. Influence of nutrition on the production and physiology of sectors produced by the insect pathogenic fungus Metarhizium anisopliae. FEMS Microbiol. Lett. 2005, 250, 201-207. [CrossRef] [PubMed]

27. Dawe, A.L.; Nuss, D.L. Hypoviruses and Chestnut Blight: Exploiting viruses to understand and modulate fungal pathogenesis. Annu. Rev. Genet. 2001, 35, 1-29. [CrossRef]

28. Kim, J.; Lee, J.; Yun, S.; So, K.; Ko, Y.; Kim, Y.; Park, S.; Kim, D. A mutant of the Bck1 homolog from Cryphonectria parasitica resulted in sectorization with an impaired pathogenicity. Mol. Plant Microbe Interact. 2016, 29, 268-276. [CrossRef]

29. Saravanakumar, D.; Lavanya, N.; Muthumeena, K.; Raguchander, T.; Samiyappan, R. Fluorescent pseudomonad mixtures mediate disease resistance in rice plants against sheath rot (Sarocladium oryzae) disease. Biocontrol 2009, 54, 273-286. [CrossRef]

30. Chowdhury, T.; Mian, M.; Mia, M.; Rafii, M.; Latif, M. Agro-ecological variations of sheath rot disease of rice caused by Sarocladium oryzae and DNA fingerprinting of the pathogen's population structure. Genet. Mol. Res. 2015, 14, 18140-18152. [CrossRef] 
31. Afolabi, O.O.; Peeters, K.J.; Onwughalu, J.; Oyetunji, O.; Oludare, A.; Ogunbayo, A.; Oni, F.; Höfte, M. Characterization of Sarocladium oryzae and pathogenic Fusarium species associated with sheath rot disease of rice in Mali and Nigeria. (unpublished; manuscript in preparation).

32. Bigirimana, V.; Hua, G.K.H.; Bertier, L.; Peeters, K.J.; Nyamangyoku, O.I.; Höfte, M. Sarocladium oryzae and Fusarium spp. are associated with rice sheath rot in Rwanda. (unpublished; manuscript in preparation).

33. Giraldo, A.; Gené, J.; Sutton, D.; Madrid, H.; de Hoog, G.; Cano, J.; Crous, P.; Guarro, J. Phylogeny of Sarocladium (Hypocreales). Persoonia 2015, 34, 10-24. [CrossRef] [PubMed]

34. Ghosh, M.; Amudha, R.; Jayachandran, S.; Sakthivel, N. Detection and quantification of phytotoxic metabolites of Sarocladium oryzae in sheath rot-infected grains of rice. Lett. Appl. Microbiol. 2002, 34, 398-401. [CrossRef] [PubMed]

35. Wang, C.; Butt, T.; St Leger, R. Colony sectorization of Metarbizium anisopliae is a sign of ageing. Microbiology 2005, 151, 3223-3236. [CrossRef] [PubMed]

36. Nuss, D.L. Hypovirulence: Mycoviruses at the fungal-plant interface. Nat. Rev. Microbiol. 2005, 3, 632-642. [CrossRef] [PubMed]

37. Li, L.; Pischetsrieder, M.; St Leger, R.J.; Wang, C. Associated links among mtDNA glycation, oxidative stress and colony sectorization in Metarhizium anisopliae. Fungal Genet. Biol. 2008, 45, 1300-1306. [CrossRef]

38. Heller, J.; Tudzynski, P. Reactive oxygen species in phytopathogenic fungi: Signaling, development, and disease. Annu. Rev. Phytopathol. 2011, 49, 369-390. [CrossRef]

39. García-Pedrajas, M.D.; Cañizares, M.C.; Sarmiento-Villamil, J.L.; Jacquat, A.G.; Dambolena, J.S. Mycoviruses in biological control: From basic research to field implementation. Phytopathology 2019, 109, 1828-1839. [CrossRef]

40. Brusini, J.; Robin, C. Mycovirus transmission revisited by in situ pairings of vegetatively incompatible isolates of Cryphonectria parasitica. J. Virol. Methods 2013, 187, 435-442. [CrossRef]

41. Bigirimana, V.P. Characterization of Sheath Rot Pathogens from Major Rice-Growing Areas in Rwanda; Ghent University: Gent, Belgium, 2016.

42. Sakthivel, N.; Gnanamanickam, S. Evaluation of Pseudomonas fluorescens for suppression of Sheath Rot disease and for enhancement of grain yields in rice (Oryza sativa L.). Appl. Environ. Microbiol. 1987, 53, 2056-2059. [CrossRef]

43. Perneel, M.; Heyrman, J.; Adiobo, A.; De Maeyer, K.; Raaijmakers, J.; De Vos, P.; Höfte, M. Characterization of CMR5c and CMR12a, novel fluorescent Pseudomonas strains from the cocoyam rhizosphere with biocontrol activity. J. Appl. Microbiol. 2007, 103, 1007-1020. [CrossRef]

44. Omura, S. The antibiotic cerulenin, a novel tool for biochemistry as an inhibitor of fatty acid synthesis. Bacteriol. Rev. 1976, 40, 681-697. [CrossRef] [PubMed]

45. Haeck, A.; Van Langenhove, H.; Harinck, L.; Kyndt, T.; Gheysen, G.; Höfte, M.; Demeestere, K. Trace analysis of multi-class phytohormones in Oryza sativa using different scan modes in high-resolution Orbitrap mass spectrometry: Method validation, concentration levels, and screening in multiple accessions. Anal. Bioanal. Chem. 2018, 410, 4527-4539. [CrossRef] [PubMed]

(C) 2020 by the authors. Licensee MDPI, Basel, Switzerland. This article is an open access article distributed under the terms and conditions of the Creative Commons Attribution (CC BY) license (http://creativecommons.org/licenses/by/4.0/). 